\title{
Método de Perfilhos Únicos para Estimar os Danos Causados pela Queima-da-bainha nos Componentes de Produtividade do Arroz
}

\author{
Leila Garcês de Araújo ${ }^{1}$, Anne Sitarama Prabhu ${ }^{1}$ \& Gisele Barata da Silva ${ }^{2}$ \\ ${ }^{1}$ Embrapa Arroz e Feijão, Cx. Postal 179, CEP 75375-000, Santo Antônio de Goiás, GO,

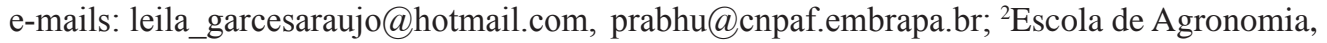 \\ Universidade Federal de Viçosa, Avenida P.H. Rolfs s/n, CEP 36570-000, Viçosa, MG, \\ e-mail: gibarata@bol.com.br
}

(Aceito para publicação em 20/01/2006)

Autor para correspondência: Anne Sitarama Prabhu

ARAÚJO, L.G., PRABHU, A.S. \& SILVA, G.B. da. Método de perfilhos únicos para estimar os danos causados pela queima-da-bainha nos componentes de produtividade do arroz. Fitopatologia Brasileira 31:199-202. 2006.

\begin{abstract}
RESUMO
O efeito da queima-da-bainha em alguns componentes da produtividade do arroz (Oryza sativa) irrigado foi estimado utilizando o método de perfilhos únicos como unidade experimental. As epidemias artificiais foram desenvolvidas através de inoculação de campo com inóculo de Rhizoctonia solani multiplicado em casca e grão de arroz. A severidade da doença foi avaliada em sete somaclones de Metica-1 e na cultivar Metica-1, considerando a altura da lesão em 50 perfilhos como variável. As relações entre o comprimento da panícula e a severidade da queima-da-bainha foram lineares e negativas para os genótipos ( $\mathrm{r}=-0.65 \mathrm{P} \leq 0.01$ ) e os de ciclo médio ( $\mathrm{r}=-0.62 \mathrm{P} \leq 0.01$ ). A queima-da-bainha afetou o comprimento da panícula em $8,83 \%$ e 6,26\% nos genótipos de ciclo precoce e médio, respectivamente. Os danos em porcentagem de espiguetas vazias para os genótipos de ciclo precoce e médio foram de 10,94\% e 12,20\%, respectivamente. A queima-da-bainha afetou mais a massa de grãos/panícula do que o enchimento das espiguetas e o comprimento da panícula, sendo de 32,75\% para os genótipos de ciclo precoce e de 30,99\% para os genótipos de ciclo médio. O método de perfilhos únicos pode ser utilizado para estimar com precisão os danos potenciais causados pela queima-da-bainha em diferentes genótipos.
\end{abstract}

Palavras-chave adicionais: Oryza sativa, epidemiologia.

\begin{abstract}
Single tillers method to estimate damage caused by sheath blight in yield components of rice

The effect of sheath blight on some yield components in irrigated rice (Oryza sativa) was estimated utilizing single tillers as experimental units. Artificial epidemics were induced by inoculating field with the inoculum of Rhizoctonia solani previously multiplied on the rice grain and hull medium. The disease severity was assessed on seven somaclones of Metica1 in addition to the parent cultivar Metica-1, considering lesion height of 50 tillers as a measure. The relationship between panicle length and sheath blight severity was linear and negative both for early precoces $(r=-0.65 \mathrm{P} \leq 0.01)$ and medium maturing ( $r=-0.62 \mathrm{P} \leq 0.01)$ ones. The disease affected panicle length by $8.83 \%$ and $6.26 \%$, and empty spikelets by $10.94 \%$ and $12.20 \%$ in early and medium maturing genotypes, respectively. The estimated mean damage in grain weight/panicle was greater than on the empty spikelets and panicle length of genotypes tested, being $32.75 \%$ for early maturing and $30.99 \%$ for late maturing ones. The single tillers method can be used to obtain precise estimates of the potential losses caused by sheath blight in different genotypes.
\end{abstract}

Additional keywords: Oryza sativa, epidemiology.

A queima-da-bainha causada por Rhizoctonia solani Kuhn [Teleomorfo Thanatephorus cucumeris (A.B. Frank) Donk] pertencente ao grupo de anastomose AG-1 IA, vem assumindo importância econômica nas várzeas tropicais de arroz (Oryza sativa L.). A alta adubação nitrogenada, a elevada densidade de plantas e a suscetibilidade das cultivares são fatores responsáveis pela alta severidade da doença em diferentes países (Gangoapadhyay \& Chakrabarti, 1982; Dath, 1990; Lee \& Rush, 1992; Groth et al., 1992), inclusive no Brasil (Prabhu et al., 2002; Rodrigues et al., 2003). Um outro fator importante para o aumento da incidência desta enfermidade é a rotação da cultura do arroz com a cultura da soja (Van Eeckhout et al., 1991; Rodrigues et al., 2003). A maioria das cultivares de arroz e de soja é suscetível à queima-da-bainha, consequentemente, a densidade de inóculo no solo aumenta ao longo dos anos com a sucessão dessas culturas (Groth et al., 1992). Atualmente, a doença ocorre, em maior ou menor grau de severidade, em todas as lavouras de arroz irrigado no Estado do Tocantins.

No Japão, ocorrem danos que variam de 24.000 a 38.000 toneladas de arroz anualmente (Gangoapadhyay \& Chakrabarti, 1982). Segundo Kozaka (1970), danos de 
$25 \%$ em produtividade são comuns quando a doença se estende até a folha bandeira e de $30 \%$ a $40 \%$ no caso de infecção severa na bainha e nas folhas. Nas Filipinas, danos na produtividade de até $24 \%$ foram relatadas em cultivares suscetíveis sob condições de alta adubação nitrogenada. Tsai (1974) em Taiwan, constatou danos de 43,0\% e 22,3\% quando a infecção teve início aos 60 dias após o plantio e no estádio de emborrachamento, respectivamente, em condições experimentais realizadas com inoculação artificial. A queima-da-bainha altera o equilíbrio de síntese e da decomposição de pigmentos de clorofila nas folhas e taxa de fotossíntese (Naidu et al., 1981). Nos estudos realizados em Beamount, Texas, E.U.A., durante cinco anos consecutivos, foram relatados danos variando de 19\% a 41\% (Marchetti \& Bollich, 1991).

Informações sobre os danos causados pelo patógeno na produtividade são importantes para racionalizar o uso de fungicidas no manejo da queima-da-bainha. No Brasil, o potencial do patógeno $R$. solani em causar danos na produtividade em arroz irrigado é desconhecido. A avaliação de danos em condições naturais de infecção no campo é dificultada devido à ocorrência de outras doenças, simultaneamente nos colmos de arroz. Fried et al. (1981), King (1976) e Rees et al. (1981) descreveram um método para análise de danos causados por doenças utilizando o perfilho como unidade experimental. As correlações entre as estimativas feitas com o método de perfilhos únicos e parcela como unidade experimental foram significativas em trigo afetado com septoriose, ferrugem das folhas e ferrugem amarela (Shtienberg et al., 1990).

O presente estudo foi realizado para estimar o potencial de danos causados pela queima-da-bainha no comprimento de panícula, na porcentagem de espiguetas vazias e na massa de grãos/panícula utilizando o método de perfilhos únicos em diferentes genótipos de arroz irrigado.

Foram utilizados oito genótipos, sendo sete somaclones de Metica-1 (quatro de ciclo precoce e três de ciclo médio) e a cultivar Metica-1 (ciclo médio), dentro de um total de 38 genótipos incluídos em um experimento para avaliação da queima-da-bainha através de inoculações artificiais em condições de campo.

Foi utilizado o delineamento inteiramente casualizado com três repetições, consistindo cada unidade experimental de cinco linhas de 3,0 $\mathrm{m}$ de comprimento espaçadas de 0,2 m. O isolado 4F1 de $R$. solani foi obtido de colmos da cultivar Metica- 1 com sintomas típicos de queima-da-bainha, oriundos de lavouras de arroz do projeto Rio Formoso, no Estado do Tocantins. O meio de cultura de casca e grão de arroz (CGA) foi usado para produção e multiplicação do inóculo. O meio CGA foi preparado misturando-se os grãos e as cascas de arroz na proporção de 3:1 (v/v) em água, em frascos Erlenmeyers com capacidade de $500 \mathrm{ml}$ e autoclavados por duas horas.

As plantas no campo foram inoculadas com $100 \mathrm{~g}$ do meio CGA colonizado pelo fungo ao redor dos perfilhos das plantas, nas três linhas centrais da parcela, aos 52 dias de idade. A severidade da doença foi avaliada com base na altura da lesão em 50 perfilhos, na linha central da parcela, na época da colheita. A severidade (\%) foi calculada considerando a altura da lesão em relação a altura total do perfilho, até o ponto de inserção da folha bandeira.

Para o estabelecimento das relações entre a severidade de queima-da-bainha e os componentes de produtividade foi utilizada uma amostra composta de 600 perfilhos, com panículas coletadas em quatro genótipos precoces e em quatro genótipos de ciclo médio (150 perfilhos de cada genótipo) nas três repetições. Os perfilhos coletados nas parcelas foram classificados de acordo com a severidade (altura da lesão em relação a altura total do perfilho) 0-20, 21 40, 41-60, 61-80 e 81-100\% de colmo afetado, considerando 50 perfilhos por repetição e tratamento. A freqüência de perfilhos por tratamento e repetição de acordo com a classe são apresentados na Tabela 1.

TABELA 1 - Número de perfilhos de arroz (Oryza sativa) de acordo com o valor de classe da severidade da queima-da-bainha causada por Rhizoctonia solani

\begin{tabular}{|c|c|c|c|c|c|c|}
\hline \multirow{2}{*}{ Genótipos } & \multirow{2}{*}{ Repetição } & \multicolumn{5}{|c|}{$\begin{array}{c}\text { Intervalo de classe de severidade } \\
\text { da queima-de-bainha }(\%)^{1}\end{array}$} \\
\hline & & $\begin{array}{l}0-20 \\
(10)^{2}\end{array}$ & $\begin{array}{c}20-40 \\
(30)\end{array}$ & $\begin{array}{c}40-60 \\
(50)\end{array}$ & $\begin{array}{c}60-80 \\
(70)\end{array}$ & $\begin{array}{c}80-100 \\
(90)\end{array}$ \\
\hline \multicolumn{7}{|l|}{ Ciclo precoce } \\
\hline SCMet 34 & 1 & 0 & 9 & 10 & 10 & 21 \\
\hline SCMet 34 & 2 & 0 & 13 & 6 & 4 & 27 \\
\hline SCMet 34 & 3 & 0 & 6 & 8 & 3 & 28 \\
\hline SCMet 04 & 1 & 4 & 17 & 11 & 3 & 15 \\
\hline SCMet 04 & 1 & 5 & 22 & 7 & 3 & 13 \\
\hline SCMet 04 & 2 & 6 & 25 & 3 & 3 & 13 \\
\hline Metica-1 & 2 & 10 & 19 & 15 & 3 & 3 \\
\hline Metica-1 & 3 & 2 & 25 & 2 & 2 & 19 \\
\hline Metica-1 & 1 & 5 & 25 & 1 & 2 & 17 \\
\hline SCMet 38 & 3 & 0 & 14 & 13 & 6 & 17 \\
\hline SCMet 38 & 2 & 9 & 13 & 9 & 3 & 15 \\
\hline SCMet 38 & 3 & 0 & 26 & 14 & 3 & 7 \\
\hline \multicolumn{7}{|l|}{ Ciclo médio } \\
\hline SCMet 04 & 1 & 10 & 17 & 9 & 4 & 10 \\
\hline SCMet 04 & 2 & 1 & 20 & 10 & 4 & 15 \\
\hline SCMet 04 & 3 & 2 & 15 & 9 & 1 & 23 \\
\hline SCMet 05 & 1 & 7 & 22 & 5 & 7 & 9 \\
\hline SCMet 05 & 2 & 4 & 16 & 8 & 8 & 14 \\
\hline SCMet 05 & 3 & 4 & 25 & 3 & 3 & 15 \\
\hline SCMet 25 & 1 & 2 & 25 & 7 & 3 & 13 \\
\hline SCMet 25 & 2 & 3 & 15 & 3 & 3 & 26 \\
\hline SCMet 25 & 3 & 2 & 14 & 8 & 2 & 24 \\
\hline Metica-1 & 1 & 4 & 27 & 23 & 2 & 3 \\
\hline Metica-1 & 2 & 1 & 13 & 9 & 4 & 23 \\
\hline Metica-1 & 3 & 2 & 13 & 3 & 1 & 31 \\
\hline
\end{tabular}

${ }^{1}$ As médias de severidade de queima-da-bainha foi calculada pela fórmula $\operatorname{Sev}(\%)=\sum$ (valor da classe $\mathrm{x}$ freqüência da classe) / número de perfilhos (50). As severidades foram de 54,13\% e 55,68\% para os genótipos de ciclo precoce e médio, respectivamente.

${ }^{2}$ Valor da classe. 
O método de perfilhos únicos e o modelo de ponto crítico foram utilizados para estimar os danos nos seguintes componentes de produtividade: comprimento de panícula, porcentagem de espiguetas vazias e massa de grãos/panícula. Foi utilizado o valor central da classe de severidade como variável independente e o de cada componente de produtividade como variável dependente na análise de regressão. O potencial de dano na produtividade, para cada componente, foi estimado com base na equação, $\hat{y}$ = a-bx, onde 'a' representou o componente de produtividade na ausência de doença e ' $b$ ' o coeficiente de regressão da taxa de redução do componente de produtividade com uma unidade de aumento da doença. $\mathrm{O}$ dano em cada componente foi calculado com a seguinte fórmula: Dano $(\%)=(a-\hat{y}) 100 /$ a, onde ' $a$ ' representou o componente de produtividade sem doença, e y o componente de produtividade estimada pela equação. As equações de regressão foram feitas para cada grupo de genótipo (ciclo precoce e médio).

Os sintomas da doença manifestaram-se aproximadamente dez dias após a inoculação dos colmos e bainhas apresentando-se como lesões ovais, elípticas, arredondadas ou irregulares. Não houve incidência de outras doenças de arroz nas condições em que o experimento foi realizado. O comprimento da panícula dos genótipos de ciclo precoce e médio diferiram significativamente pelo teste de $\mathrm{t}(\mathrm{t}=8,66 \mathrm{P} \leq 0,01)$. As severidades foram calculadas com base na altura de lesão em relação a altura total. As relações entre o comprimento da panícula e a severidade da queimada-bainha foram lineares e negativas para os genótipos de ciclo precoce e médio (Figura 1). O comprimento da panícula diminuiu em $0,4 \mathrm{~cm}$ para cada $1 \%$ de severidade de queima-da-bainha para os genótipos de ciclo precoce e de 0,3 cm para ciclo médio.

Os danos, em porcentagem de espiguetas vazias, calculadas com base nas equações de regressão linear, para os genótipos precoces e de ciclo médio foram de 52,0\% e $52,4 \%$, respectivamente. A severidade média da doença foi de 54,13\% para os genótipos de ciclo precoce e de 55,68\% para os genótipos de ciclo médio, em campo. O dano médio estimado na massa de grãos/panícula foi de $32,75 \%$ para os genótipos de ciclo precoce e de 30,99\% para os genótipos de ciclo médio (Tabela 2). A queima-da-bainha afetou mais a massa de grãos/panícula do que o enchimento das espiguetas e o comprimento da panícula.

As estimativas baseadas em perfilhos individuais
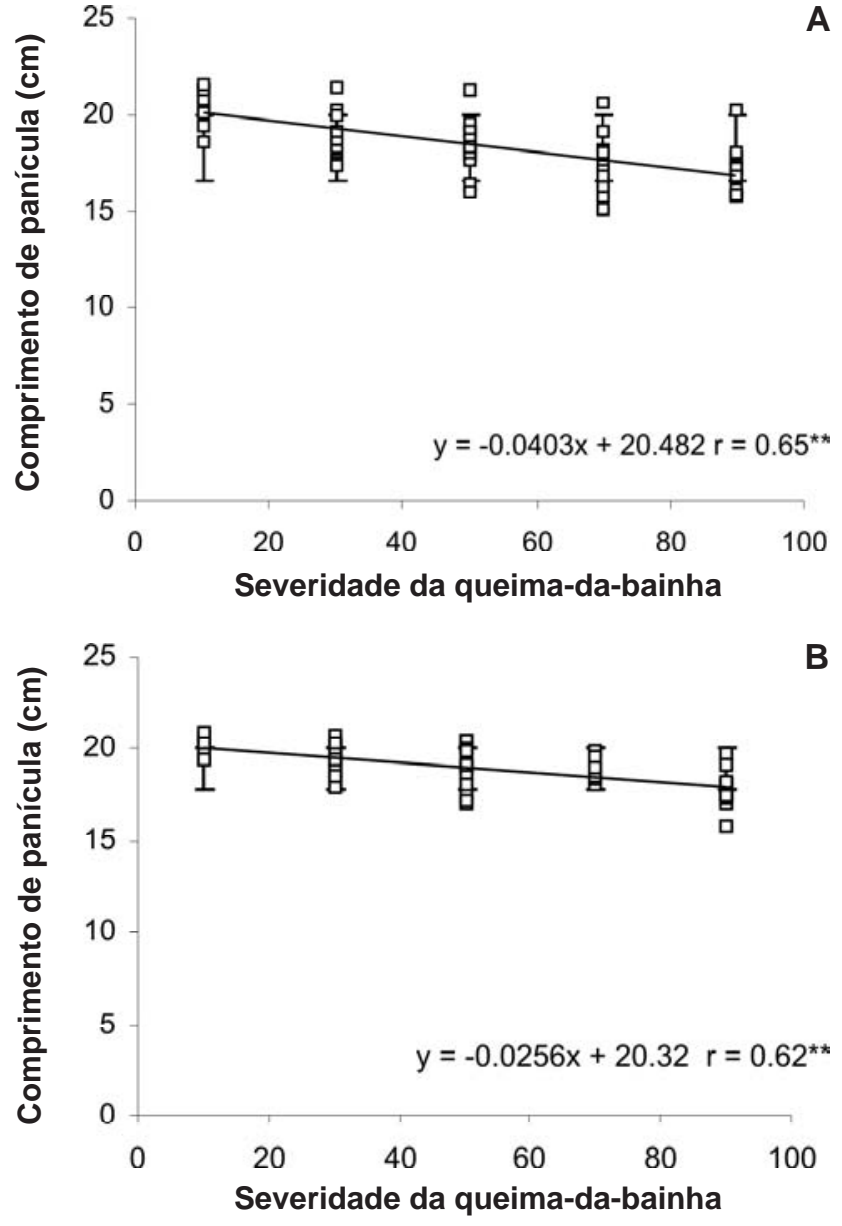

FIG. 1 - Relação entre comprimento de panícula de arroz (Oryza sativa) e severidade de queima-da-bainha causada por Rhizoctonia solani. A. quatro genótipos de ciclo precoce; B. quatro genótipos de ciclo médio. As equações foram baseadas nos dados conjunto de repetições e genótipos.

podem ser similares às das parcelas. Este método tem valor quando a doença não afeta o número de perfilhos (James \& Teng, 1979). No presente estudo as inoculações foram realizadas aos 52 dias após o plantio e não afetou o perfilhamento.

Os resultados da presente investigação mostraram que os danos nos diferentes componentes da produtividade podem ser quantificadas com o método de perfilhos únicos

TABELA 2 - Danos nos componentes de produtividade devido à queima-da-bainha causada por Rhizoctonia solani em oito genótipos de arroz (Oryza sativa)

\begin{tabular}{|c|c|c|c|c|c|c|}
\hline \multirow{2}{*}{ Componentes de produtividade } & \multicolumn{3}{|c|}{ Genótipos de ciclo precoce ${ }^{1}$} & \multicolumn{3}{|c|}{ Genótipos de ciclo médio ${ }^{1}$} \\
\hline & Equações de regressão $^{2}$ & $\mathbf{r}$ & Dano (\%) & Equações de regressão $^{3}$ & $r$ & $\operatorname{Dano}(\%)$ \\
\hline Comprimento de panícula & $\hat{y}=-0,0332 x+20,352$ & $0,98^{* *}$ & 8,83 & $\hat{\mathrm{y}}=-0,0291 \mathrm{x}+20,347$ & $0,98 * *$ & 6,26 \\
\hline Porcentagem de espiguetas vazias & $\hat{y}-0,2022 x+10,487$ & $0,97^{* *}$ & 10,94 & $\hat{y}-0,2192 x+12,25$ & $0,97^{* *}$ & 12,20 \\
\hline Massa de grãos/panícula & $\hat{y}=-0,0129 x+2,1305$ & $0,99 * *$ & 32,75 & $\hat{y}=-0,0095 x+1,7066$ & $0,99 * *$ & 30,99 \\
\hline
\end{tabular}


e com equações de regressão simples. Este método pode ser estendido para estimar os danos causados pela queima-dabainha nos componentes de produtividade em lavouras de arroz.

O efeito da doença somente no estádio final do crescimento foi relacionado com alguns componentes de produtividade utilizando o modelo de ponto crítico (James \& Teng, 1979). Este método de avaliação somente em um estádio facilita a coleta simultânea de dados de doença e de panículas com grãos formados nas lavouras de produtores para estimar os danos potenciais, independentemente do ciclo da cultivar. Quando ocorre mais de uma doença os danos podem ser determinadas através da regressão múltipla utilizando as severidades das diferentes doenças como variáveis independentes e os componentes de produtividade como variáveis dependentes. O agrupamento de perfilhos de acordo com o nível de severidade da doença permitiu aumentar os valores de $\mathrm{R}^{2} \mathrm{e}$ a validação destes modelos pode ser feita em lavouras comerciais.

\section{REFERÊNCIAS BIBLIOGRÁFICAS}

DATH, A.P. Sheath blight disease of rice and its management. New Delhi. Associated Publishing Company. 1990.

FRIED, P.M., MACKENZIE, D.P. \& NELSON, R.R. Yield loss caused by Erysiphe graminis f. sp. tritici on single culms of 'Chacellort' wheat and four multilines. Z. Pflanzenkrankh. Pflanzenschutz 88:256-264. 1981.

GANGOAPADHYAY, S. \& CHAKRABARTI, N.K. Sheath blight of rice. Review of Plant Pathology 61:451-460. 1982.

GROTH, D.E., RUSH, M.C. \& HOLLIER, C.A. Prediction of rice sheath blight severity and yield loss based on early season infection. Louisiana Agriculture 35:20-23. 1992.

JAMES, W.C. \& TENG, P.S. The quantification of production associated with plant disease. Applied Biology 4:21-267. 1979.

KING, J.E. Relationship between yield loss and severity of yellow rust recorded on large number of single stems of wheat. Plant Pathology 25:172-177. 1976.

KOZAKA, T. Pellicularia sheath blight of rice plants and its control. Japanese Agricultural Research Quarterly 5:12-16. 1970.

LEE, F.N. \& RUSH, M.C. Rice sheath blight: a major rice disease. Plant Disease 67:829-832. 1992.

MARCHETTI, M.A \& BOLLICH, C.N. Quantification of the relationship between sheath blight severity and yield loss in rice. Plant Disease 75:773-775. 1991.

NAIDU, D.V., RAO, B.S. \& MURTY, P.S.S. Influence of sheath blight infection on the leaves of chlorophyll and ${ }^{14} \mathrm{CO}_{2}$ uptake in rice. Indian Phytopathology 34:30-33. 1981.

PRABHU, A.S., FILIPPI, M.C.; SILVA, G.B. \& SANTOS, G.R. Resistência de cultivares de arroz a Rhizoctonia solani e Rhizoctonia oryzae. Pesquisa Agropecuária Brasileira 37:589-595. 2002.

REES, R.G.R., MAYER, J. \& PLAZ, G.J. Yield loss in wheat from yellow spot: a disease loss relationship derived from single tillers. Australian Journal of Agricultural Research 32:851-859. 1981.

RODRIGUES, F.A., VALE, F.X.R., KORNDÖRFER, G.H., PRABHU, A.S., DATNOFF, L.E., OLIVEIRA, A.M.A. \& ZAMBOLIM, L. Influence of silicon on sheath blight of rice in Brazil. Crop Protection 22:23-29. 2003.

SHTIENBERG, A., DINOOR, A. \& MARANI, A. Evaluation of the single tillers method for yield loss assessment in wheat under Israel conditions. Phytopathology 130:331-341. 1990.

TSAI, W.H. Assessment of yield losses due to sheath blight at different inoculation stages. Journal of Taiwan Agricultural Research 23:88-194. 1974.

VAN EECKHOUT, E., RUSH, M.C. \& BLACKWELL, M. Effect of rate and timing of fungicide application on incidence and severity of sheath blight and grain yield of rice. Plant Disease 75:1254-1261. 1991. 